\title{
Detection of DTI White Matter Abnormalities in Multiple Sclerosis Patients
}

\author{
Olivier Commowick ${ }^{1}$, Pierre Fillard ${ }^{2}$, Olivier Clatz $^{2}$, and Simon K. Warfield ${ }^{1}$ \\ ${ }^{1}$ Computational Radiology Laboratory, Department of Radiology, \\ Children's Hospital, 300 Longwood Avenue, Boston, MA, 02115, USA \\ Olivier.Commowick@childrens . harvard.edu \\ ${ }^{2}$ INRIA Sophia Antipolis - Asclepios Team, 2004 Rte des Lucioles BP 93 \\ 06902 Sophia Antipolis Cedex, France
}

\begin{abstract}
The emergence of new modalities such as Diffusion Tensor Imaging (DTI) is of great interest for the characterization and the temporal study of Multiple Sclerosis (MS). DTI indeed gives information on water diffusion within tissues and could therefore reveal alterations in white matter fibers before being visible in conventional MRI. However, recent studies generally rely on scalar measures derived from the tensors such as FA or MD instead of using the full tensor itself. Therefore, a certain amount of information is left unused.

In this article, we present a framework to study the benefits of using the whole diffusion tensor information to detect statistically significant differences between each individual MS patient and a database of control subjects. This framework, based on the comparison of the MS patient DTI and a mean DTI atlas built from the control subjects, allows us to look for differences both in normally appearing white matter but also in and around the lesions of each patient. We present a study on a database of 11 MS patients, showing the ability of the DTI to detect not only significant differences on the lesions but also in regions around them, enabling an early detection of an extension of the MS disease.
\end{abstract}

\section{Introduction}

Multiple sclerosis (MS) causes a demyelination of the white matter fibers. The origin and evolution of this disease are still not well understood, and numerous studies have been conducted to evaluate its evolution and its influence on neighboring brain structures. To this end, several methods have been proposed, mainly using conventional MR modalities like T1, FLAIR or T2 images to delineate lesions as in [1. This task is challenging and crucial, as having access to the lesion load over time, coupled with measurements such as local brain atrophy, can bring a lot of information on the characterization of the disease.

The emergence of new modalities, and in particular diffusion tensor imaging (DTI) 2, is of great interest in MS. DTI gives information about water diffusion within tissues, and could therefore reveal alterations in normal appearing white matter fibers before being visible in conventional MRI. For this purpose,

D. Metaxas et al. (Eds.): MICCAI 2008, Part I, LNCS 5241, pp. 975-982. 2008.

(C) Springer-Verlag Berlin Heidelberg 2008 
Filippi et al. [3] evaluated the differences in fractional anisotropy (FA) between MS patients and controls on normal appearing white matter regions. While this type of studies tend to show that diffusion MRI could be beneficial to detect more accurately MS lesions, they generally rely on scalar measures derived from the tensor such as FA or mean diffusivity, instead of using the full tensor itself. A certain amount of information is thus lost in the processing of this data. In parallel, other studies have shown the interest of using the whole tensor information. For example, 4] showed that it was possible to compare diffusion tensors of two populations using Log-Euclidean (LE) metrics [5], and that the comparison was more sensitive, i.e. could reveal more differences, than considering only a scalar parameter such as the FA.

In this work, we propose a framework that relies on the whole diffusion tensor information to detect statistically significant differences between MS patients and controls. Recent work proposed methods for the detection of group differences between two tensor populations such as 4 on diffusion tensors, or 6 on Jacobian tensors derived from deformation fields. However, in our context, these methods are not directly applicable as lesions are not spatially consistent. Consequently, it is necessary to compare each patient individually with the group of control subjects. This approach also raises the question of the construction of an unbiased reference frame from the controls.

To address those problems, we propose a framework to compute an unbiased atlas of diffusion tensors from a database of controls. Based on this atlas, we show how to compute statistical differences between a patient and the atlas using a z-score derived from the whole tensor.

This framework was evaluated on a database of 11 MS patients and 31 normal controls, showing significant differences between each individual patient and the controls in regions identified as lesions on the T1 and FLAIR images. Interestingly, our results shows that DTI can remain sensitive inside a thick ribbon surrounding the lesions, suggesting that DTI could be more sensitive than conventional MRI to detect MS lesions.

\section{Materials and Methods}

\subsection{Construction of a Diffusion Tensor Atlas of Controls}

In order to compare the DTI of normal controls with the patient DTI, we need to bring all the DTI into the same reference frame. Using any subject as a reference would introduce a bias due to the specific anatomy of this reference image. Therefore, we chose to build a geometrically unbiased atlas from the dataset of controls extending the work of [7], and use this atlas as the reference for the rest of the study.

Our algorithm basically iterates over two steps until convergence (in our application the average image was built in 6 iterations). In a first step, all images $I_{k}$ are non linearly registered onto an initial (randomly chosen) reference image $R$, using a block-matching based non linear registration method presented in 8]. These registrations produce the deformation fields $T_{k}$. A mean image $M$ is 
built from all registered images by averaging them. In a second step, the deformation fields $T_{k}$ are averaged to get a mean transformation $\bar{T}$, which inverse is applied to $M$. This new mean image is then used as the new reference image for the next iteration, i.e. $R=M \circ \bar{T}^{-1}$. Notice that we used the Log-Euclidean framework for diffeomorphisms [9] when averaging deformation fields to ensure the invertibility of $\bar{T}$. Transformations are calculated based on T1 images and then applied on DTIs, the main reason being that DTI registration is still not a mature research topic.

When warping diffusion tensor images, special attention must be paid to the reorientation of tensors. Indeed, tensors are supposed to remain aligned with underlying tissues under any transform. We chose the finite strain reorientation strategy proposed in [10] to reorient the tensors with respect to the Jacobian of the deformation. Note that the principal direction of diffusion preserving method [11] could be used as well. Tensors are resampled and averaged using Log-Euclidean metrics as they provide a fast way to extend any interpolation method to tensors while preserving the positive-definite constraint, and without suffering from the swelling effect as this is the case of the Euclidean calculus.

In summary, a set of transformations $T_{k}$ is obtained for each T1 image $I_{k}$ along with a mean T1 image $\bar{I}$. Each $T_{k}$ is applied to its corresponding DTI. The DTI atlas is finally generated by averaging these images.

\subsection{Evaluation of Statistical Differences between Atlas and Patient}

In statistics, differences between a test data and a population are estimated through the Mahalanobis distance, also abusively called z-score in this article by analogy to the univariate z-test. This distance requires to know the covariance matrix of the population (of tensors in our case). We propose to detail its computation in a first step, and show in a second step how to extract a p-value from it to test for significance.

Covariance Matrix and z-Score. The covariance matrix is computed as follows. Tensor logarithms are turned into vectors by keeping only the 6 independent coefficients and multiplying off-diagonal elements by $\sqrt{2}$, so that the $L_{2}$ norm of tensors is compatible with the $L_{2}$ norm of vectors. We call this operation the $V e c$ mapping. Then, the covariance matrix $C$ of this 6 -dimensional vector is calculated. Finally, the Mahalanobis distance between a tested tensor $\tilde{D}$ and the ensemble mean/covariance matrix of the atlas is given by:

$$
z(\tilde{D})=\left(\tilde{D}_{L o g}-\bar{D}_{L o g}\right)^{\top} C^{-1}\left(\tilde{D}_{L o g}-\bar{D}_{L o g}\right)
$$

where $\tilde{D}_{L o g}$ and $\bar{D}_{L o g}$ are the $V e c$ mappings of the tensor logarithms, i.e. $\tilde{D}_{L o g}=$ $\operatorname{Vec}(\log (\tilde{D}))$.

Computing the p-Value Associated to the z-Score. In our case, the null hypothesis is that the diffusion tensor being tested belongs to the normal population. As in the scalar case, the p-value is given by $P\left(X>I_{c}(z)\right)$, where $I_{c}(z)$ is 
defined as the points $x$ where $r \equiv\left(x-\bar{D}_{L o g}\right)^{\top} C^{-1}\left(x-\bar{D}_{L o g}\right)=z^{2}$. In our specific context, the probability density function to be integrated is a $6 \mathrm{D}$ multivariate Gaussian, leading to the following formulation:

$$
V(z)=1-P\left(X<I_{c}(z)\right)=1-\int_{r<z^{2}} G_{\bar{D}_{L o g}, C}(x) d x
$$

where $G$ is a multivariate Gaussian of mean $\bar{D}_{\text {Log }}$ and covariance matrix $C$. It can be shown that this integration is equivalent to the integration of a multivariate Gaussian of mean 0 and covariance $I d$ for values of $x$ such as $x^{\top} x<z^{2}$. Using this simplification and a change of coordinates to spherical coordinates, we obtain the following expression for the p-value:

$$
V(z)=e^{-\frac{z^{2}}{2}}\left(1+\frac{z^{2}}{2}+\frac{z^{4}}{8}\right)
$$

Following the usual practice in voxel-based morphometry, average p-values are computed over spatially coherent regions of interest $R$ (for instance MS lesions). To this end, we have chosen to compute first a mean z-score over the voxels $i$ in region $R$ and use it to compute the p-value, i.e. $\bar{V}_{R}=V\left(\frac{1}{N} \sum_{i \in R} z\left(\tilde{D}_{i}\right)\right)$.

\section{Results}

\subsection{Image Databases}

Data. For this study, we used a dataset of 31 controls and 11 patients diagnosed with MS. For each control, a T1 image and a DTI were acquired. T1 images have a resolution of $256 \times 256 \times 176$ and a voxel size of $1 \times 1 \times 1 \mathrm{~mm}^{3}$. DTI images were acquired with 12 gradients and have a resolution of $256 \times 256 \times 60$ and a voxel size of $1 \times 1 \times 2.5 \mathrm{~mm}^{3}$.

For each patient, a T1 image (resolution $124 \times 256 \times 256$, voxel size $1.3 \times$ $\left.1 \times 1 \mathrm{~mm}^{3}\right)$ and a DTI $(12$ gradients, resolution $256 \times 256 \times 60$, voxel size $1 \times 1 \times 2.5 \mathrm{~mm}^{3}$ ) were also available, as well as T2 and FLAIR images.

Pre-Processing. For each control and each patient, the image sequence (DTI, FLAIR, T2) was rigidly registered onto the T1 image. For the DTI, the B0 image was rigidly registered on the T1 image, and the obtained transform was applied to the diffusion gradients. To correct for acquisition distortion in DTI, we applied a non rigid registration procedure between the $\mathrm{B} 0$ of the DTI acquisition and the T1 image using the B-Splines method of 12 with few control points $(5 \times 5 \times 5)$. B0 to $\mathrm{T} 1$ registration results were visually validated by a radiologist. $\mathrm{T} 1$ and FLAIR images were finally used to manually delineate the MS lesions on each patient.

For each MS patient, non linear registration of the T1 image onto the atlas was done. Patient to atlas registration consists in two steps. First, a global affine transformation was computed between the T1 images. Then, non linear registration was performed to locally align the anatomies of the atlas and the patient. As intensities in voxels with lesions are different from those of the atlas, 


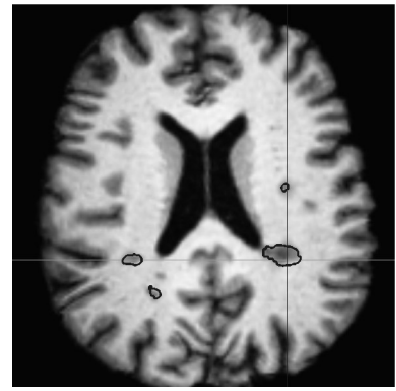

(a)

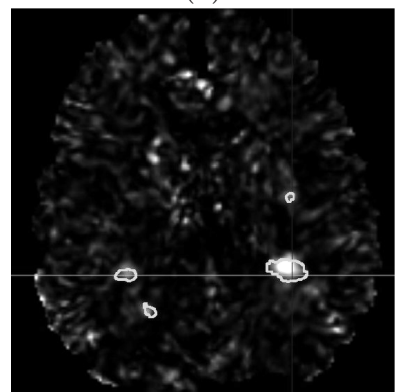

(d)

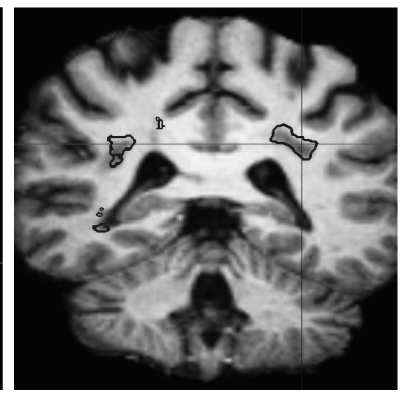

(b)

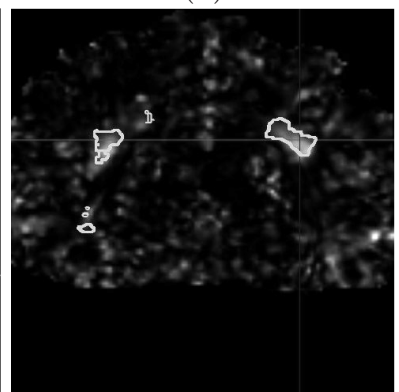

(e)

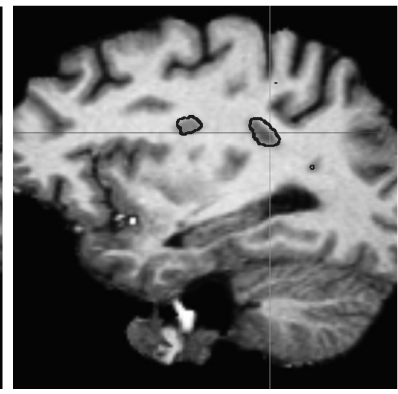

(c)

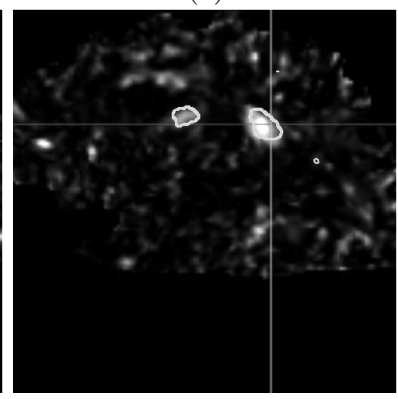

(f)

Fig. 1. Voxel-wise z-scores for a given MS patient. Illustration of the z-score maps obtained for a patient (images (d), (e), (f)) compared to the corresponding anatomy (axial (a),(d), coronal (b),(e) and sagittal slices (c),(f)). The contours correspond to the manual delineations of the MS lesions.

the transformation was interpolated inside a dilated mask of the patient's lesions following a method similar to [13. Thus, abnormalities in lesions signal did not interfere during the registration process. The final transformations were then applied to the patient DTI as presented in Sec. 2.1. Finally, voxel-wise z-score and $\mathrm{p}$-value calculation were done to obtain a map of statistical differences. Results are presented in the next section.

\subsection{Diffusion Tensor Differences in MS Lesions}

The initial evaluation consisted in looking at differences between patients and controls in the lesion regions. We present in Fig. 1 the maps of z-scores, with the manual delineations of the lesions superimposed, for one patient of the database.

This figure shows that MRI regions with visible lesions have the highest zscores. This indicates that the diffusion tensor is affected by MS and that lesions are highly likely to be significantly different with respect to an atlas of controls. We can also see on this figure (particularly on the sagittal slice) that, for some lesions, the manual delineation does not correspond exactly to the high z-score values. This is mainly caused by local errors in manual delineation, as shown in the sagittal view of Fig. 1 (c) at the position of the axes. More interestingly, 
Table 1. Quantitative evaluation results on the MS lesions. Mean p-values obtained on regions corresponding to the MS lesions for each patient.

\begin{tabular}{|c|c||c|c||c|c|}
\hline Patient $\#$ & p-value & Patient $\#$ & p-value & Patient $\#$ & p-value \\
\hline 1 & $2.1 \times 10^{-18}$ & 5 & $4.2 \times 10^{-7}$ & 9 & $8.7 \times 10^{-4}$ \\
\hline 2 & $7.9 \times 10^{-5}$ & 6 & $7.5 \times 10^{-5}$ & 10 & $6.8 \times 10^{-4}$ \\
\hline 3 & 0.045 & 7 & $3.4 \times 10^{-4}$ & 11 & $4.2 \times 10^{-7}$ \\
\hline 4 & $4.9 \times 10^{-4}$ & 8 & $1.8 \times 10^{-7}$ & & \\
\hline
\end{tabular}

the map of z-scores presents hyper-signals in regions not labeled as lesion by the expert. For instance, a possible extension of a lesion can be seen on the sagittal slice. There are indeed high z-score values around the large lesion on the right of this view that are not seen in the T1 image.

To verify and quantify the DTI signal difference between the patients and the control subjects, we computed the mean tensor-based p-value inside the manually delineated lesions. Results are presented in Table 1

All p-values are below the critical value of 0.05 , therefore rejecting for all patients the null hypothesis: tensors in lesions are not likely to belong to the normal variability represented by the atlas of controls. Interestingly, patient 3 is closer to 0.05 than the other patients.

\subsection{Evaluation of Tensor Differences Around Lesions}

The previous section has shown the existence of significant tensor-based differences between patients and controls inside the lesions. To go beyond this sensitivity analysis, we looked at surrounding regions to check whether regions around the lesions exhibit large z-score values.

These ring-like regions around the lesions were obtained by subtracting the binary mask of the manually segmented lesions to its dilation. P-values were then computed withing those regions surrounding the lesions. These results are presented in Table 2 ,

Results show, for 7 patients out of 11, a p-value that is below the threshold of 0.05 . Remaining p-values are also very low (the maximum value being 0.101 ). This may suggest that some patients exhibit an extension of the lesions that is not visible in conventional MRI, but is detectable in DTI using higher order statistical analysis. These results tend to prove that DTI may effectively be used

Table 2. Quantitative evaluation results on regions around the MS lesions. Mean p-values obtained on regions around the MS lesions for each patient (see text).

\begin{tabular}{|c|c||c|c||c|c|}
\hline Patient $\#$ & $\mathrm{p}$-value & Patient $\#$ & $\mathrm{p}$-value & Patient $\#$ & $\mathrm{p}$-value \\
\hline 1 & $8.1 \times 10^{-7}$ & 5 & 0.009 & 9 & $1.9 \times 10^{-3}$ \\
\hline 2 & 0.059 & 6 & $1.8 \times 10^{-3}$ & 10 & 0.028 \\
\hline 3 & 0.101 & 7 & 0.079 & 11 & 0.019 \\
\hline 4 & 0.066 & 8 & 0.016 & & \\
\hline
\end{tabular}


as a complementary diagnostic imaging modality to study and understand the evolution of MS.

\section{Conclusion}

We have presented in this article a new framework for the detection of significant differences between DTI of patients and normal controls. This framework was applied to the study of multiple sclerosis and highlighted the great interest of DTI measurements made on the whole tensor for a better understanding and characterization of the disease. Results indeed indicated, qualitatively and quantitatively, that diffusion tensors were not only significantly different inside the lesion regions, but also for some patients in regions around the lesions. This suggests the existence of an extension of the disease invisible in conventional MRI, but revealed by DTI.

This comparison framework is however dependent on the choice of the registration method to build and register the statistical atlas. This choice may indeed bias the p-values results. The comparison of different registration methods will therefore be interesting to quantify this dependency. This evaluation will also include the use of registration methods taking into account the specificities of the DTI such as [14], allowing to register more precisely regions such as the white matter, which has a uniform intensity in conventional MRI.

Another way to cope with this bias and possible preprocessing errors is the study of robust analysis methods, including more information on the local neighborhood of the tensors. An interesting solution could be the use of the STAPLE method [15] on each of the tensors components, allowing to compute at the same time a robust mean tensor image and error parameters of the patient tensors.

This work also opens wide and important perspectives for the understanding of multiple sclerosis. One of them will be the temporal study of the evolution of the lesions and the comparison of the invisible extents found through DTI with the real evolution of the disease. This will allow us to see if these extents effectively evolve into lesions or if they are more related to a local edema. This framework will also be used for the evaluation of the influence of the disease on normal appearing white matter regions such as the corticospinal tracts.

In the future, we would also like to perform a quantitative comparison of all available imaging modalities to independently detect and quantify the lesion volume in the brain. Finally, as an extension to the present work, it will be interesting to propose a multi modal statistical framework to compute statistics on multivariate data (i.e. DTI, FLAIR, T1, T2) of higher dimensions. Indeed, this statistical study will also allow to do a sensitivity analysis of the contribution of each modality in the final detection performance.

\section{Acknowledgments}

This investigation was supported in part by a research grant from CIMIT, grant RG 3478A2/2 from the NMSS, and by NIH grants R03 CA126466, R01 RR021885, R01 GM074068 and R01 EB008015. 


\section{References}

1. Ge, Y.: Multiple sclerosis: The role of MR imaging. American Journal of Neuroradiology 27, 1165-1176 (2006)

2. Basser, P., Mattiello, J., Bihan, D.L.: MR diffusion tensor spectroscopy and imaging. Biophysical Journal 66, 259-267 (1994)

3. Filippi, M., Cercignani, M., Inglese, M., Comi, M.H.G.: Diffusion tensor magnetic resonance imaging in multiple sclerosis. Neurology 56, 304-311 (2001)

4. Whitcher, B., Wisco, J.J., Hadjikhani, N., Tuch, D.S.: Statistical group comparison of diffusion tensors via multivariate hypothesis testing. Magnetic Resonance in Medicine (57), 1065-1074 (2007)

5. Arsigny, V., Fillard, P., Pennec, X., Ayache, N.: Log-Euclidean metrics for fast and simple calculus on diffusion tensors. Magnetic Resonance in Medicine 56(2), 411-421 (2006)

6. Lepore, N., Brun, C.A., Chiang, M.C., Chou, Y.Y., Lopez, O.L., Aizenstein, H.J., Toga, A.W., Becker, J.T., Thompson, P.M.: Multivariate statistics of the jacobian matrices in tensor based morphometry and their application to HIV/AIDS. In: Larsen, R., Nielsen, M., Sporring, J. (eds.) MICCAI 2006. LNCS, vol. 4190, pp. 191-198. Springer, Heidelberg (2006)

7. Guimond, A., Meunier, J., Thirion, J.P.: Average brain models: A convergence study. Computer Vision and Image Understanding 77(2), 192-210 (2000)

8. Commowick, O., Malandain, G.: Evaluation of atlas construction strategies in the context of radiotherapy planning. In: Proc. of the SA2PM Workshop, Copenhagen. Held in conjunction with MICCAI 2006 (October 2006)

9. Arsigny, V., Commowick, O., Pennec, X., Ayache, N.: A Log-Euclidean framework for statistics on diffeomorphisms. In: Larsen, R., Nielsen, M., Sporring, J. (eds.) MICCAI 2006. LNCS, vol. 4190, pp. 924-931. Springer, Heidelberg (2006)

10. Ruiz-Alzola, J., Westin, C.F., Warfield, S.K., Alberola, C., Maier, S., Kikinis, R.: Nonrigid registration of 3D tensor medical data. MedIA 6(2), 143-161 (2002)

11. Alexander, D., Pierpaoli, C., Basser, P., Gee, J.: Spatial transformations of diffusion tensor magnetic resonance images. IEE TMI 20(11), 1131-1139 (2001)

12. Rueckert, D., Sonoda, L.L., Hayes, C., Hill, D.L.G., Leach, M.O., Hawkes, D.J.: Nonrigid registration using free-form deformations: Application to breast MR images. IEEE Transactions on Medical Imaging 18(8), 712-721 (1999)

13. Stefanescu, R., Commowick, O., Malandain, G., Bondiau, P.Y., Ayache, N., Pennec, $\mathrm{X}$.: Non-rigid atlas to subject registration with pathologies for conformal brain radiotherapy. In: Barillot, C., Haynor, D.R., Hellier, P. (eds.) MICCAI 2004. LNCS, vol. 3216, pp. 704-711. Springer, Heidelberg (2004)

14. Goodlett, C., Davis, B., Jean, R., Gilmore, J.H., Gerig, G.: Improved correspondence for DTI population studies via unbiased atlas building. In: Larsen, R., Nielsen, M., Sporring, J. (eds.) MICCAI 2006. LNCS, vol. 4191, pp. 260-267. Springer, Heidelberg (2006)

15. Warfield, S.K., Zou, K.H., Wells, W.M.: Validation of image segmentation by estimating rater bias and variance. In: Larsen, R., Nielsen, M., Sporring, J. (eds.) MICCAI 2006. LNCS, vol. 4191, pp. 839-847. Springer, Heidelberg (2006) 